\title{
Variación en los patrones de crecimiento previo a la muerte de individuos de Austrocedrus chilensis a lo largo de un gradiente pluviométrico en el norte de la Patagonia Andina
}

\author{
Leticia Vega ${ }^{1,2, \mathbb{1}}$; Eugenia Marcotti ${ }^{2 / 3}$ \& Mariano M. Amoroso ${ }^{4,5}$ \\ ${ }^{1}$ Facultad de Ciencias Agrarias, Universidad Nacional de Cuyo. Mendoza, Argentina. ${ }^{2}$ Instituto Argentino de Nivología \\ y Glaciología, Consejo Nacional de Investigaciones Científicas y Técnicas. Mendoza, Argentina. ${ }^{3}$ Instituto de Ecorregiones \\ Andinas - Jujuy. ${ }^{4}$ Centro Científico Tecnológico Patagonia Norte, Consejo Nacional de Investigaciones Científicas y Técnicas. \\ ${ }^{5}$ Instituto de Investigaciones en Recursos Naturales, Agroecología y Desarrollo Rural, Universidad Nacional de Río Negro. Río \\ Negro, Argentina.
}

\begin{abstract}
Resumen. Los bosques Andino-Patagónicos albergan una de las coníferas endémicas de América del Sur: Austrocedrus chilensis (D. Don) Pic. Ser. et Bizzarri (Cupressaceae). El hecho de que ocurran eventos episódicos de mortalidad a lo largo del gradiente de distribución de la especie hace necesarios estudios que echen luz sobre el comportamiento de los árboles en tales eventos. El objetivo de este trabajo fue estudiar los patrones de crecimiento previo a la muerte de individuos de Austrocedrus chilensis en un gradiente de precipitación O-E. El área comprende la distribución septentrional de la especie en el norte de la Patagonia argentina. Se seleccionaron nueve sitios de muestreo a lo largo de una transecta O-E en las cercanías del Lago Traful, provincia de Neuquén. La transecta abarca un gradiente de precipitación entre 1800 y $600 \mathrm{~mm} /$ año. Se analizaron patrones y tasas de crecimiento en los 5, 10 y 15 años previos a la muerte a lo largo del gradiente. Los resultados permitieron establecer que, según la variabilidad de los patrones de crecimiento, los sitios analizados se ordenaron en dos grandes grupos según el tipo de bosque: mixtos-compactos y compactos-ecotonales. A su vez, estas diferencias se relacionaron con condiciones particulares de las cronologías (e.g., el índice de ancho de anillo) y biofísicas (e.g., pendiente, exposición, latitud y longitud) de los sitios de estudio. Las tasas de crecimiento previas a la muerte permitieron determinar que, en todos los sitios de estudio, los individuos presentaron una disminución en el crecimiento radial previo a este evento; a su vez, para iguales ventanas temporales, los individuos muertos crecieron menos que los individuos vivos. En base a estos resultados, es necesario profundizar los estudios para conocer los mecanismos por los que se ordenan estos patrones de crecimiento previo a la muerte a lo largo del gradiente Andino-Patagónico.
\end{abstract}

[Palabras clave: mortalidad, gradiente de precipitación]

Aвstract. Variation in the growth patterns prior to death of individuals of Austrocedrus chilensis along a pluviometric gradient in the North of Andean Patagonia. The Andean-Patagonian forests are home to one of the endemic conifers of South America: Austrocedrus chilensis (D. Don) Pic. Ser. Et Bizzarri (Cupressaceae). The occurrence of episodic mortality events along the gradient of distribution of this species makes necessary studies to shed light on allow advance on the responses of trees to these tree behaviors in these events. The objective of this research is to study of the growth patterns prior to the death of individuals of Austrocedrus chilensis in a W-E precipitation gradient. Specifically, the area includes the northern distribution of the species in northern Patagonia, Argentina. Nine sampling sites were selected along a west-east transect in the vicinity of Traful Lake, Neuquén province, which captures a precipitation gradient of 1800 to $600 \mathrm{~mm} /$ year. Patterns and growth rates were analyzed in the 5,10 and 15 years prior to death along the gradient. The results obtained allowed to establish that according to the variability of the growth patterns ordered in two analyzed forests were ordered in two large groups: mixed-compact and compact-ecotonal. In turn, these differences were related to particular conditions of the chronologies (e.g., the ring width index) and biophysics (e.g., slope, exposure, latitude and longitude) of the study sites. The rates of growth prior to death allowed to determine that in all the study sites, individuals presented a decrease in radial growth prior to this event; in turn, for the same time windows, dead individuals grew less than living individuals. It is necessary to deepen the studies to know the mechanisms by which these growth patterns are ordered prior to death along the Andean-Patagonian gradient.

[Keywords: mortality, precipitation gradient]

Editor asociado: Ricardo Grau 


\section{INTRODUCCIÓN}

A escala global, muchas especies arbóreas experimentan procesos de mortalidad masivas y episódicas. Por ello, en ecosistemas forestales de todo el mundo se están estudiando los patrones de mortalidad (e.g., Villalba and Veblen 1998; Adams et al. 2009; Amoroso et al. 2015; Cailleret et al. 2017). Como consecuencia de estos procesos de mortalidad tiene lugar la pérdida de hábitat de especies silvestres, la oportunidad para que sucedan invasiones de especies exóticas, la formación de nuevas comunidades y la interrupción temporal de la provisión de algunos bienes y servicios ecosistémicos (McKenney 2007; Adams et al. 2009; Matías 2012). Además, los episodios extensos de mortalidad pueden impactar en los balances de carbono (C), lo que reduce el potencial de secuestro en los ecosistemas e incrementa las pérdidas de C a través de los suelos. Esto, sumado a la potencialidad de liberaciones de $\mathrm{C}$ a la atmósfera por episodios de mortalidad de gran amplitud podría contribuir de manera negativa al cambio climático global (Adams et al. 2009).

Diversos autores sostienen que los eventos climáticos (e.g., sequías extremas), que pueden interactuar con agentes biológicos o procesos denso-dependientes, serían la causa principal de los procesos de mortalidad (Camarero et al. 2012; Ruiz-Benito 2013; Cailleret et al. 2017). A pesar de esto, la comprensión de los mecanismos fisiológicos que conducen a la mortalidad y los cambios asociados en las tasas de crecimiento de los árboles previo a la muerte sigue siendo limitada, así como la capacidad para predecir la mortalidad y sus impactos en el tiempo y en el espacio (Bigler et al. 2007; Cailleret et al. 2017). A su vez, muchos de los estudios se realizaron en ecotonos (Allen and Breshears 1998; Villalba and Veblen 1998; Suárez and Kitzberger 2008), y dejaron de lado otros ambientes o ecosistemas de la distribución de las especies. Por esta razón aumentó la demanda de estudios demográficos en ecosistemas boscosos (incluyendo los extremos de la distribución de las especies) o en contraste de condiciones abióticas (e.g., a lo largo de gradientes) con el fin de mejorar la comprensión de los ecosistemas y su sensibilidad frente a los cambios futuros en la variabilidad climática (Suarez and Kitzberger 2008).

El análisis del crecimiento de los árboles mediante el ancho de los anillos se usó con éxito para reconstruir patrones de crecimiento y mortalidad, para estudiar respuestas a estrés ambiental y para evaluar el crecimiento previo a la muerte (Pedersen 1998; Bigler et al. 2007; Allen et al. 2010; Amoroso et al. 2015). Los datos de resolución anual obtenidos mediante los métodos dendrocronológicos facilitan el análisis retrospectivo de muchos procesos ecológicos. Esto permite estudiar respuestas y patrones en escalas temporales y espaciales amplias, que de otra manera demandarían un seguimiento prolongado en el tiempo y a escalas espaciales menores (Amoroso and Suárez 2015).

En América del Sur, los bosques andinopatagónicos constituyen una franja cordillerana angosta donde se encuentra una de las coníferas endémicas de Chile y Argentina, Austrocedrus chilensis (D. Don) Pic. Ser. et Bizzarri (Cupressaceae). En el contexto de los futuros cambios ambientales, la vulnerabilidad de los bosques de $A$. chilensis aumentó durante las últimas décadas debido a procesos de decaimiento, cambios en el uso de la tierra, tala y disturbios varios (e.g., incendios) (Suárez and Amoroso 2013; González et al. 2014). La ocurrencia de eventos episódicos de mortalidad a lo largo del amplio gradiente de distribución de la especie hace necesaria una visión regional que permita dar cuenta de las diferencias que ocurren entre los patrones de crecimiento previo a la muerte de los individuos creciendo en distintas condiciones ambientales a lo largo del gradiente. Este trabajo propone estudiar y caracterizar los patrones de crecimiento radial previo a la muerte y su relación con las condiciones biofísicas de los sitios, a lo largo del gradiente pluviométrico en el que se distribuye $A$. chilensis.

\section{Materiales y MÉTOdos}

\section{Área de estudio y muestreo a campo}

A. chilensis, llamado localmente ciprés de la cordillera, es la conífera nativa de mayor rango latitudinal del Bosque Subantártico de Chile y Argentina. En nuestro país se extiende de forma discontinua. Presenta su extremo más septentrional a los $37^{\circ} 07^{\prime} \mathrm{S}$ y $70^{\circ} 36^{\prime} \mathrm{O}$ en la provincia de Neuquén (Bran et al. 2002; Pastorino et al. 2006) y el más austral a los $43^{\circ} 44^{\prime} \mathrm{S}$ y $71^{\circ} 23^{\prime} \mathrm{O}$ en la provincia de Chubut (Pastorino and Gallo 2002; Pastorino 2015).

El área de distribución de $A$. chilensis se encuentra bajo la influencia de la circulación atmosférica del oeste, que determina un flujo 
casi permanente de masas de aire húmedo desde el Océano Pacífico (Garreaud et al. 2009). La orientación N-S de la Cordillera de Los Andes determina una barrera para estas masas de aire húmedo que provienen del Pacífico (Bianchi 2016). Debido a esto, las poblaciones de $A$. chilensis se ubican en un gradiente de precipitación marcado, con dirección O-E, que se extiende desde la zona cordillerana con $1800 \mathrm{~mm}$ anuales hasta el ecotono con la estepa patagónica, donde la precipitación es $600 \mathrm{~mm}$ anuales (La Manna 2005).

En la zona más húmeda se encuentran los 'bosques mixtos', donde $A$. chilensis codomina el dosel arbóreo con especies del género Nothofagus. Hacia el este, en la zona media del gradiente, se encuentran rodales monoespecíficos que constituyen los 'bosques compactos', y en el ecotono con la estepa, zona más xérica, $A$. chilensis se encuentra en pequeños grupos formando los 'bosques marginales o ecotonales' (Dezzoti and Sancholuz 1991; Gallo and Pastorino 2004; Pastorino 2015).

El área de estudio se encuentra en el extremo norte de la distribución de la especie, al suroeste de la provincia de Neuquén, en el área de Confluencia-Traful, dentro del Parque Nacional Nahuel Huapi. Para obtener muestras representativas de los diferentes ambientes se seleccionaron nueve sitios de muestreo a lo largo de una transecta de $\sim 50$ $\mathrm{km}$ en la cuenca del lago Traful $\left(40^{\circ} \mathrm{S}-71^{\circ} 42^{\prime}\right.$
O y $40^{\circ} \mathrm{S}-71^{\circ} 60^{\prime} \mathrm{O}$ ) (Figura 1). Los muestreos se realizaron en noviembre de 2013 y octubre de 2015. En cada sitio se estableció una parcela con el objetivo de muestrear al menos 12 individuos muertos. El tamaño de cada parcela varió según la cantidad y la densidad de individuos muertos que se encontraron en cada rodal. Se evitó seleccionar aquellos sitios que presentaban síntomas de mortalidad por decaimiento forestal (Amoroso et al. 2015), como así también los sitios con evidencias de agentes de disturbios como fuego o viento, que podrían haber ocasionado la mortalidad.

Para garantizar la presencia de corteza o del último anillo de crecimiento, de cada individuo muerto se tomó un mínimo de dos muestras de barreno a la altura del pecho. Además, para complementar la estimación de la fecha de muerte se realizaron observaciones morfológicas referidas al porcentaje de corteza presente y la presencia o ausencia de ramas en el árbol. Estas características permiten tener una idea de cuán reciente es la muerte del individuo, y aportan información extra para utilizar durante la etapa del fechado. Por ejemplo, la presencia de corteza y ramas en un individuo muerto podría indicar que su muerte fue reciente; en cambio, su ausencia se podría relacionar con una muerte más antigua (Pastorino et al.,2015).

En cada parcela se registraron las coordenadas geográficas y la altitud. La pendiente y la exposición de los sitios se obtuvieron con el software QGIS utilizando el Modelo Digital
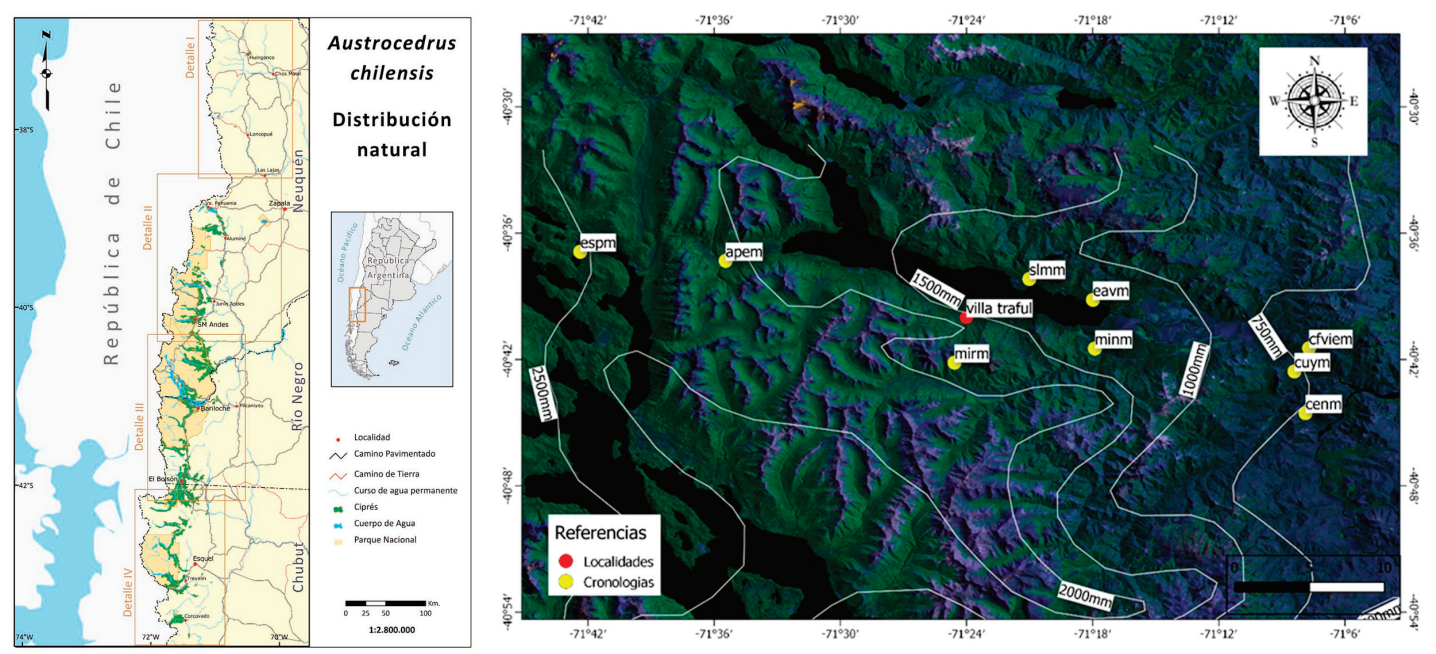

Figura 1. Área de distribución de los bosques de A. chilensis y área de estudio en el recuadro rojo (imagen de la izquierda), extraído de Pastorino 2015. A la derecha se indican los sitios de muestreo (ver tabla 1 por el detalle de los códigos).

Figure 1. A. chilensis forest distribution and study area in the red box (image on the left), from Pastorino 2015. On the right, sampling sites are indicated (see table 1 for the detail of the codes). 
de Elevaciones (DEM) para el área (Tabla 1). Los valores de precipitación media anual para los diferentes sitios de muestreo se estimaron a partir de las isohietas obtenidas por Cordón y colaboradores (1993). El tipo de bosque se determinó en base a las características estructurales siguiendo las clasificaciones de Dezzoti y Sancholuz (1991) y Pastorino y colaboradores (2015).

\section{Trabajo de laboratorio}

Las muestras fueron procesadas por medio de métodos dendrocronológicos estándares (Stockes and Smiley 1968) y fueron fechadas de manera visual bajo lupa binocular (50x). La datación se inició desde la corteza hacia la médula. El datado visual se hizo por medio de la técnica skeleton plot (Stockes and Smiley 1968). El ancho de los anillos de crecimiento se midió en dirección paralela a los tejidos radiales, desde la médula hacia la corteza, en una tableta Velmex UniSlide conectada a un contador digital Metronics Quick-Chek QC$10 \mathrm{~V}$, con una precisión de $0.001 \mathrm{~mm}$. El datado de las series de anillos de individuos muertos fue asistido por comparación con cronologías de ancho de anillos maestras de individuos vivos de la especie, existentes para la zona (Villalba and Veblen 1996; Amoroso et al. 2015). La calidad del fechado visual se evaluó con el programa COFECHA (Holmes 1983; Grissino-Mayer 2001), que permitió establecer el año calendario correcto del último anillo de crecimiento completo. Además, se corrigieron los errores relacionados con anillos ausentes, falsos y 'paquetes de anillos'.

La fecha de mortalidad de cada árbol se estableció como el último anillo presente (completo o incompleto) previo a la corteza, a través del fechado de estas muestras con las cronologías de árboles vivos. En este proceso resultaron clave los datos que se obtuvieron a campo, como el porcentaje de corteza y la presencia o ausencia de ramas, ya que aportaron información para la determinación final de la fecha de mortalidad.

\section{Construcción de las cronologías de ancho de anillos}

Para cada sitio se ajustó a todas las series individuales un modelo horizontal a la media. Este ajuste correspondiente al crecimiento medio no altera las tendencias presentes en las series originales y proporciona a cada árbol un mismo peso en la cronología (Kitzberger et al. 2000). También contribuye a maximizar la señal relacionada con disturbios endógenos y exógenos en el rodal, lo que permite comparar y conocer la dinámica de la mortalidad de individuos de $A$. chilensis en el área de estudio. Las series estandarizadas para cada individuo se combinaron para obtener una cronología de ancho de anillos por sitio. Para esto, se utilizó el paquete dpl R (Bunn 2008; Rodríguez Catón 2014) del software libre $R$.

\section{Análisis de los patrones de crecimiento}

Para los individuos muertos se calculó el incremento medio anual (IMA) del ancho de anillo utilizando los datos de las cronologías crudas (raw) para cada sitio. Con el objetivo de comparar el crecimiento promedio de los individuos muertos entre los sitios, los valores medios de IMA de ancho de anillo se compararon utilizando un ANOVA no paramétrico y se usó la prueba de Kruskal Wallis (García-González et al. 1997).

Para conocer la relación entre los patrones de crecimiento previo a la muerte de los individuos entre los diferentes sitios se calculó el coeficiente de correlación de Spearman

Tabla 1. Características de los sitios de muestreo. Los códigos de los sitios figuran de oeste a este según la ubicación en el gradiente.

Table 1. Characteristics of the sampling sites. Site codes are presented from west to east depending on the location on the gradient.

\begin{tabular}{|c|c|c|c|c|c|c|c|c|}
\hline Código & Nombre & $\begin{array}{l}\text { Latitud } \\
\left(^{\circ}\right)\end{array}$ & $\begin{array}{c}\text { Longitud } \\
\left({ }^{\circ}\right)\end{array}$ & $\begin{array}{l}\text { Altitud } \\
\text { (m.s.n.m) }\end{array}$ & Exposición & $\begin{array}{c}\text { Pendiente } \\
\left({ }^{\circ}\right)\end{array}$ & $\begin{array}{l}\text { Precipitación } \\
\text { media anual } \\
(\mathrm{mm})\end{array}$ & $\begin{array}{l}\text { Tipo de } \\
\text { bosque }\end{array}$ \\
\hline ESPM & Lago Espejo & -40.6147 & -71.7061 & 881.70 & $238.72(\mathrm{SO})$ & 9.67 & 2000 a 2500 & Mixto \\
\hline APEM & Arroyo Pedregoso & -40.6219 & -71.5908 & 853.80 & $357.51(\mathrm{NO})$ & 13.07 & 2000 a 2500 & Mixto \\
\hline SLMM & Sendero Las Mellizas & -40.6362 & -71.3500 & 788.00 & $254.24(\mathrm{SO})$ & 20.08 & 1000 a 1500 & Compacto \\
\hline EAVM & Estancia Arroyo Verde & -40.6527 & -71.2997 & 637.00 & $175.23(\mathrm{SE})$ & 23.34 & 1000 a 1500 & Compacto \\
\hline MIRM & Mirador & -40.7025 & -71.4094 & 912.80 & $278.97(\mathrm{NO})$ & 25.03 & 1500 a 2000 & Compacto \\
\hline MINM & Río Minero & -40.6913 & -71.2982 & 852.60 & $44.52(\mathrm{NE})$ & 45.05 & 1000 a 1500 & Compacto \\
\hline CUYM & Cuyín Manzano & -40.7094 & -71.1400 & 815.00 & 50.63 (NE) & 24.68 & 750 a 1000 & Ecotonal \\
\hline CENM & Centinela & -40.7424 & -71.1311 & 786.00 & 115.62 (SE) & 31.85 & 750 a 1000 & Ecotonal \\
\hline CFVM & Confluencia-Paso del viento & -40.6910 & -71.1284 & 709.40 & $318.14(\mathrm{NO})$ & 30.41 & 750 a 1000 & Ecotonal \\
\hline
\end{tabular}


para un período temporal común. El período común utilizado fue 1866-1948, para el cual todas las cronologías presentaron cinco o más series.

Cabe destacar que las correlaciones de Spearman se realizaron utilizando las cronologías residuales a fin de disminuir los efectos de la alta autocorrelación observada en todas las cronologías, de acuerdo con la metodología empleada en otros estudios con la misma y con otras especies (Mundo et al. 2011). La autocorrelación corresponde al coeficiente de correlación entre una serie y esta misma desfasada un año (-1), por lo cual provee una estimación de cómo el crecimiento de un año está influenciado por el crecimiento del año anterior (Rodríguez Catón 2014).

Por otro lado, se realizó un análisis de componentes principales (ACP) para identificar las asociaciones entre la variabilidad del crecimiento radial previo a la muerte (valores medios de IMA de ancho de anillo de cada sitio) y las variables biofísicas (i.e., pendiente, latitud, longitud, exposición, altitud y precipitación media anual) de los diferentes sitios muestreados a lo largo del gradiente pluviométrico. Para los grupos resultantes del ACP, se realizó un ANOVA no paramétrico a través de un análisis de Kruskal Wallis para evaluar la ocurrencia o no de diferencias significativas entre los sitios según las características que las variables biofísicas presentaron en cada uno.

\section{Tasas de crecimiento previo a la muerte}

Las tasas de crecimiento previo a la muerte se calcularon utilizando los valores de ancho de anillo de las series estandarizadas, siguiendo una metodología similar a la de Amoroso y Daniels (2010). Las tasas se calcularon para distintas ventanas temporales $(5,10$ y 15 años previos a la muerte) según la siguiente fórmula:

$$
\mathrm{T}(\mathrm{i})=\frac{\mathrm{X}(\mathrm{i})-\mathrm{X}}{\mathrm{X}}
$$

donde i representa los años previos a la muerte (5, 10 y 15); T(i) es la tasa de crecimiento del período de 5, 10 ó 15, años previos a la muerte según sea i; X(i) es el promedio del índice de ancho de anillo de cada serie, para las ventanas temporales de 5, 10 ó 15 años incluyentes entre sí, y X es el promedio del índice de ancho de anillo de cada serie a lo largo de toda la vida del árbol.
Para evaluar las tasas de crecimiento previo a la muerte entre las diferentes ventanas de tiempo se realizó un ANOVA no paramétrico y una prueba de Kruskal Wallis en estos períodos para cada sitio. Como variables se emplearon los valores de las tasas de crecimiento previas a la muerte de los árboles de cada sitio; como tratamientos, las distintas ventanas temporales establecidas en cada sitio. Del mismo modo se evaluaron las tasas de crecimiento relacionadas con los dos grupos que surgieron del ACP; como variables se usaron las tasas para de crecimiento previas a la muerte en distintas ventanas temporales y como tratamientos, el grupo del ACP al que pertenece cada árbol del sitio.

Una vez determinada la fecha de muerte de cada individuo se comparó el crecimiento medio de éstos con los árboles vivos del sitio (Cailleret et al. 2017). Se evaluó el crecimiento previo a la muerte para las tres ventanas temporales (5, 10 y 15 años), en relación con el crecimiento de individuos vivos para el mismo período de tiempo, por medio de la siguiente fórmula:

$$
C(i)=\frac{X_{m}(i)}{X_{v}(i)}
$$

donde i corresponde a los años previos a la muerte $(5,10$ y 15); C es la relación entre el crecimiento previo a la muerte de cada individuo y el crecimiento promedio de la cronología de individuos vivos del mismo sitio para el mismo período; Xm es el promedio del índice de ancho de anillo de cada individuo, y $\mathrm{Xv}_{\mathrm{v}}$ es el promedio del índice de ancho de anillo de la cronología de individuos vivos para cada sitio. La relación menor o mayor a 1 significa que el crecimiento del individuo previo a su muerte fue menor o mayor que en los individuos vivos, respectivamente.

\section{Resultados}

\section{Cronologías de individuos muertos}

Las cronologías de ancho de anillo obtenidas para los distintos sitios difirieron en su longitud (número de años), período temporal comprendido (años calendario), tamaño muestral y amplitud en la variación del índice de ancho de anillo en relación con su media (Tabla 2, Figura 2). En general, las cronologías de los sitios ubicados en el ecotono con la estepa fueron de mayor longitud con respecto a las de los sitios ubicados hacia el oeste. En cuanto a la variabilidad interanual, el sitio ESPM (extremo oeste) mostró la menor 
Tabla 2. Estadísticos de las cronologías de ancho de anillo de individuos muertos para cada sitio (ver Tabla 1 para el detalle de los códigos).

Table 2. Statistics of the ring width chronologies of dead individuals for each site (see Table 1 for the details of codes).

\begin{tabular}{|c|c|c|c|c|c|c|c|c|}
\hline \multirow[b]{2}{*}{ Sitio } & \multirow[b]{2}{*}{$\mathrm{N}^{\circ}$ indiv. } & \multirow[b]{2}{*}{$\mathrm{N}^{\circ}$ series } & \multirow[b]{2}{*}{ Período } & \multicolumn{2}{|c|}{ Datos de medición } & \multirow[b]{2}{*}{$\begin{array}{c}\text { RBAR } \\
\text { total }\end{array}$} & \multicolumn{2}{|c|}{$\begin{array}{l}\text { Cronologías } \\
\text { estándar }\end{array}$} \\
\hline & & & & $\begin{array}{l}\text { IMA } \\
(\mathrm{mm})\end{array}$ & $\begin{array}{l}\text { Sensibilidad } \\
\text { media }\end{array}$ & & EPS & $\begin{array}{c}\text { Auto- } \\
\text { correlación }\end{array}$ \\
\hline ESPM & 21 & 38 & $1811-2005$ & 1.77 & 0.23 & 0.44 & 0.92 & 0.82 \\
\hline APEM & 13 & 20 & 1793-2006 & 1.54 & 0.22 & 0.21 & 0.69 & 0.83 \\
\hline SLMM & 14 & 26 & $1831-2007$ & 1.56 & 0.24 & 0.59 & 0.95 & 0.92 \\
\hline EAVM & 12 & 19 & $1835-2007$ & 1.62 & 0.24 & 0.44 & 0.85 & 0.89 \\
\hline MIRM & 18 & 31 & $1802-2009$ & 1.07 & 0.20 & 0.35 & 0.90 & 0.94 \\
\hline MINM & 6 & 10 & $1788-2012$ & 2.39 & 0.23 & 0.14 & 0.26 & 0.69 \\
\hline CUYM & 6 & 8 & $1779-2014$ & 0.57 & 0.28 & 0.53 & 0.32 & 0.73 \\
\hline CENM & 14 & 27 & $1662-2007$ & 1.20 & 0.28 & 0.47 & 0.84 & 0.81 \\
\hline CFVM & 9 & 18 & $1801-1998$ & 1.20 & 0.27 & 0.62 & 0.93 & 0.91 \\
\hline
\end{tabular}

amplitud en comparación con los otros sitios (Figura 2). El lapso común para el que las cronologías de todos los sitios contaban con cinco o más series fue de 82 años (1866 a 1948).

La sensibilidad media de las cronologías osciló entre 0.21 y 0.28 (Tabla 2), valores que se encuentran dentro del rango aceptable para la especie (Villalba and Veblen 1997; Villalba et al. 1998; Amoroso et al. 2015). En todos los sitios, los valores de autocorrelación de primer orden fueron mayores a 0.60 , lo cual también concuerda con valores reportados previamente para A. chilensis (Villalba and Veblen 1997; Villalba et al. 1998).

Los sitios ESPM, APEM, SLMM, MIRM y MINM (bosques mixtos y compactos) difirieron significativamente de EAVM y CUYM. Este último fue el de menor valor de incremento medio anual (IMA) de ancho de anillo, con $0.57 \mathrm{~mm} / \mathrm{año}$. Los sitios CENM y CFVM (bosques ecotonales) presentaron un IMA con características similares a los bosques mixtos y compactos $(\mathrm{H}=34.84, P<0.0001)$ (Figura 3).
Los análisis de correlación entre las cronologías residuales para el período común 1866-1948 resultaron positivas en todos los casos. En general, los sitios cercanos entre sí dentro del gradiente presentaron valores de correlación más altos (Tabla 3 ).

Las fechas de mortalidad variaron ampliamente en cada sitio y a lo largo del gradiente (Figura 4). En el sitio ESPM se concentraron mayormente en el primer lustro de la década del 2000, mientras que para APEM fue en dos períodos: uno, durante la década del ochenta; otro, entre 1994 y 2006. En los sitios EAVM, SLMM y MIRM, las fechas de mortalidad correspondieron a los comienzos de las décadas de 1990 y 2000 (este último para los tres sitios). En MINM y CUYM, las fechas fueron muy variables en el tiempo; para el primero tuvieron lugar en las décadas de 1980 y 2000; para el segundo, en 1887, 1908, 1953 y en la década del 2000. De manera similar, el sitio CENM en la primera década del 2000. Por último, para CFVM, los eventos de mortalidad ocurrieron durante las décadas de 1970 y 1980 (en particular, 1998).

Tabla 3. Coeficientes de correlación (Spearman). Nivel de significancia $\alpha=0.05$ (ver Tabla 1 para el detalle de los códigos).

Table 3. Correlation coefficients (Spearman). Significance level $\alpha=0.05$ (see Table 1 for the details of codes).

\begin{tabular}{cccccccccc}
\hline & ESPM & APEM & SLMM & EAVM & MIRM & MINM & CUYM & CENM & CFVM \\
\hline ESPM & 1 & & & & & & & & \\
APEM & 0.51 & 1 & & & & & & & \\
SLMM & 0.60 & 0.39 & 1 & & & & & & \\
EAVM & 0.57 & 0.55 & 0.71 & 1 & & & & \\
MIRM & 0.56 & 0.58 & 0.71 & 0.83 & 1 & & & \\
MINM & 0.41 & 0.41 & 0.48 & 0.51 & 0.61 & 1 & & \\
CUYM & 0.32 & 0.28 & 0.54 & 0.57 & 0.59 & 0.39 & 1 & \\
CENM & 0.47 & 0.39 & 0.55 & 0.65 & 0.66 & 0.59 & 0.70 & 1 \\
CFVM & 0.46 & 0.41 & 0.59 & 0.61 & 0.63 & 0.47 & 0.63 & 0.66 & 1 \\
\hline
\end{tabular}




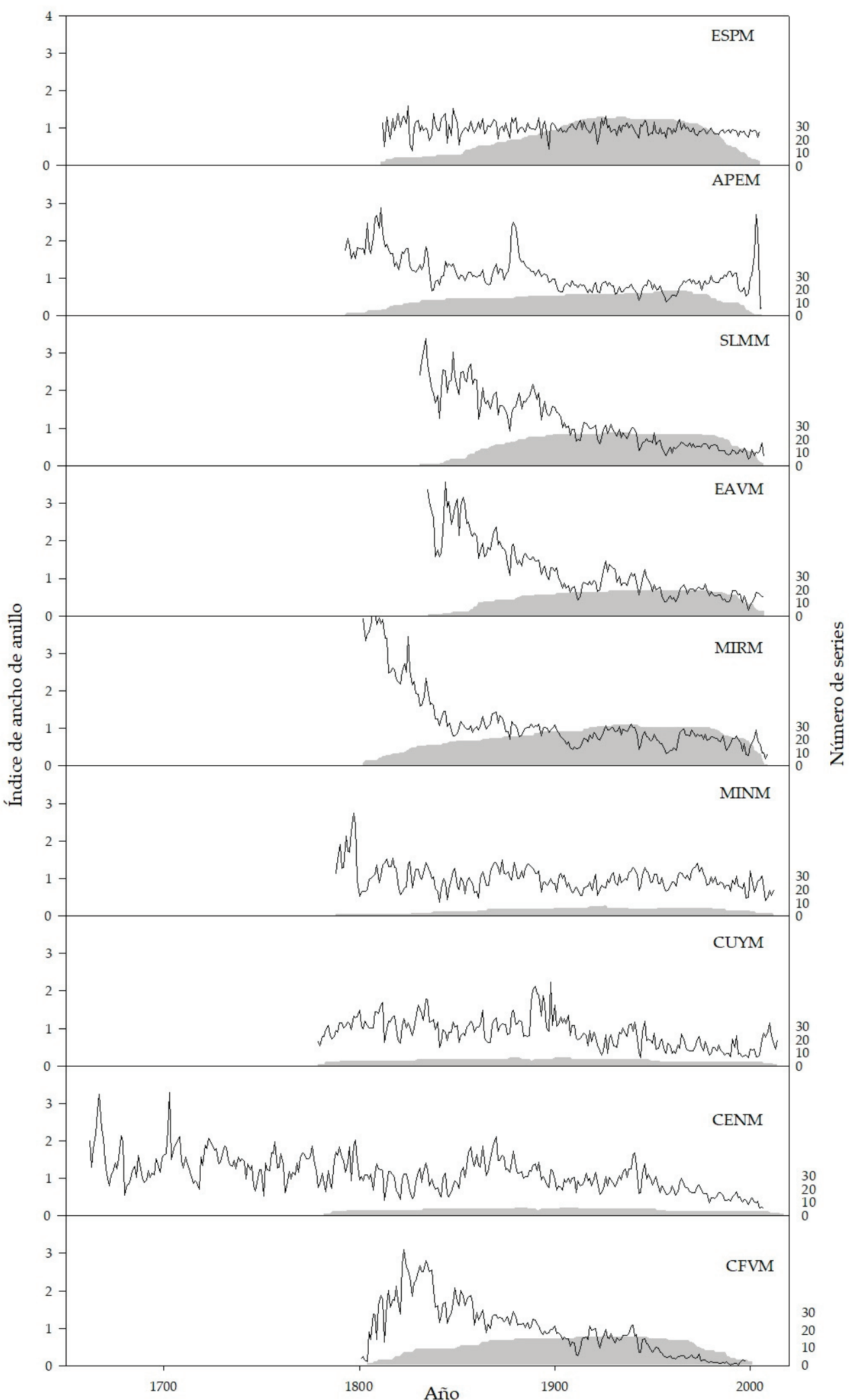

Figura 2. Cronologías de ancho de anillo estándar de A. chilensis para los sitios estudiados. El área en gris indica el número de series incluidas en cada cronología (ver tabla 1 por el detalle de los códigos).

Figure 2. Standard ring width chronologies of $A$. chilensis for the studied sites. The gray area indicates the number of series included in each chronology (see table 1 for the detail of the codes). 


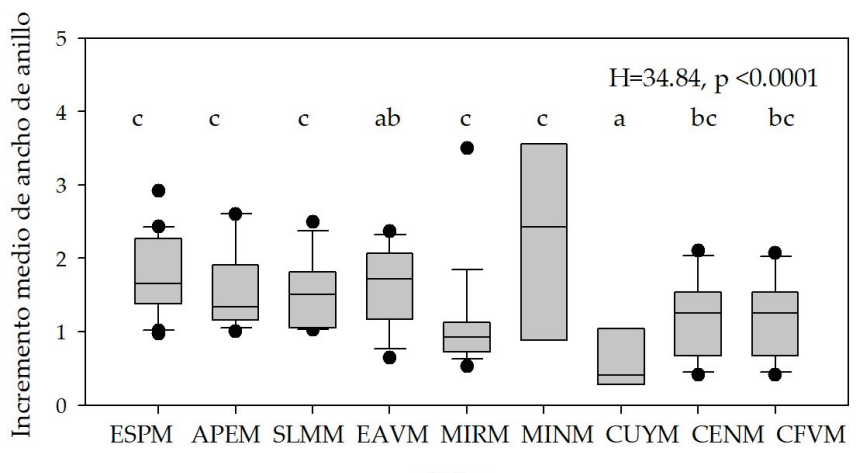

Sitios
Figura 3. Incremento medio anual (IMA) de los sitios en el gradiente, desde los bosques mixtos a ecotonales (izquierda y derecha, respectivamente) (ver tabla 1 por el detalle de los códigos).

Figure 3. Average annual Increment (IMA) of the sites along the gradient, from mixed to ecotonal forests (left and right, respectively) (see table 1 for the detail of the codes).

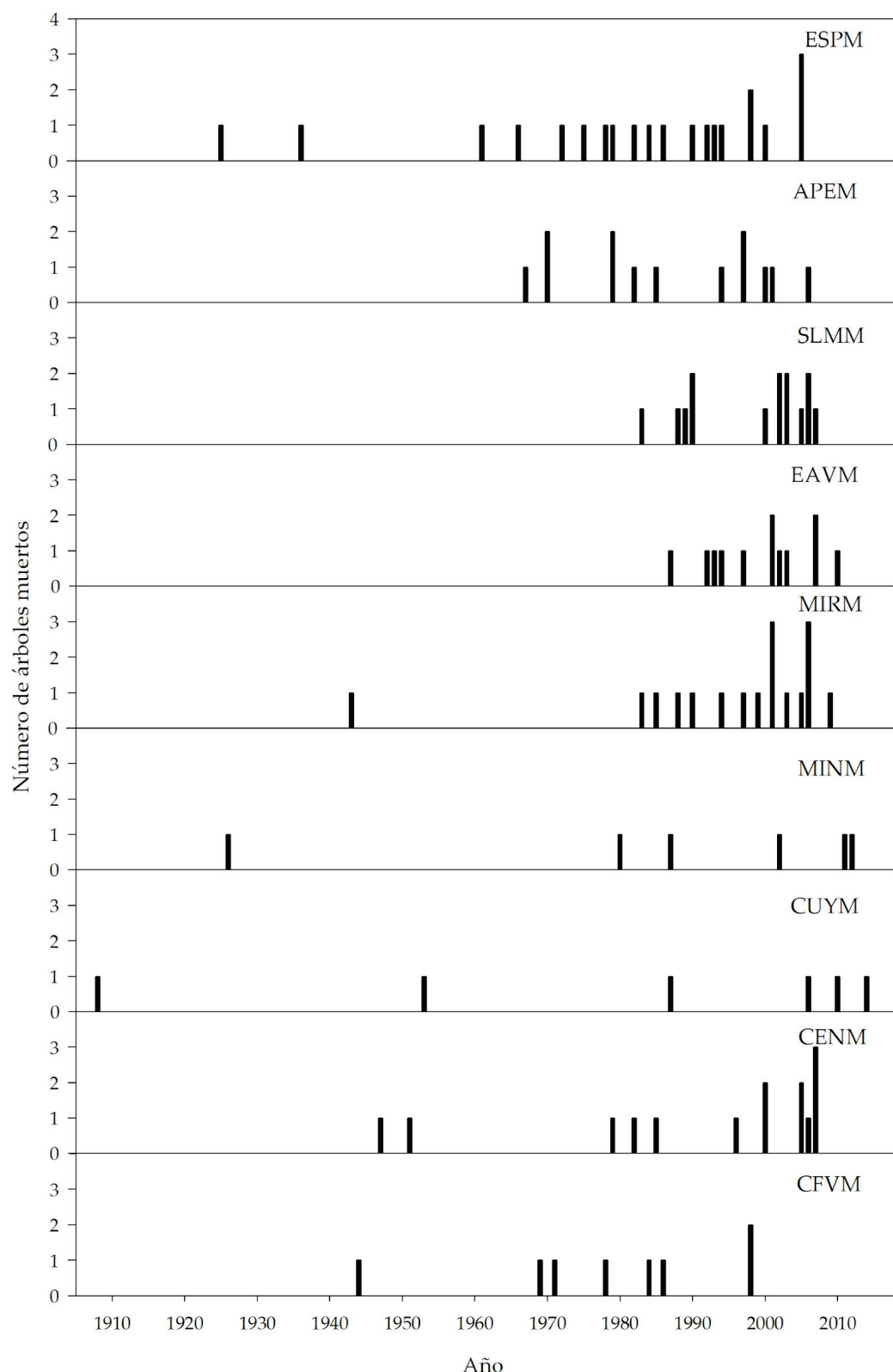

Figura 4. Fechas de muerte en el tiempo correspondientes a cada sitio a lo largo del gradiente (ver tabla 1 por el detalle de los códigos).

Figure 4. Dates of death overtime for each site along the gradient (see table 1 for the detail of the codes). 


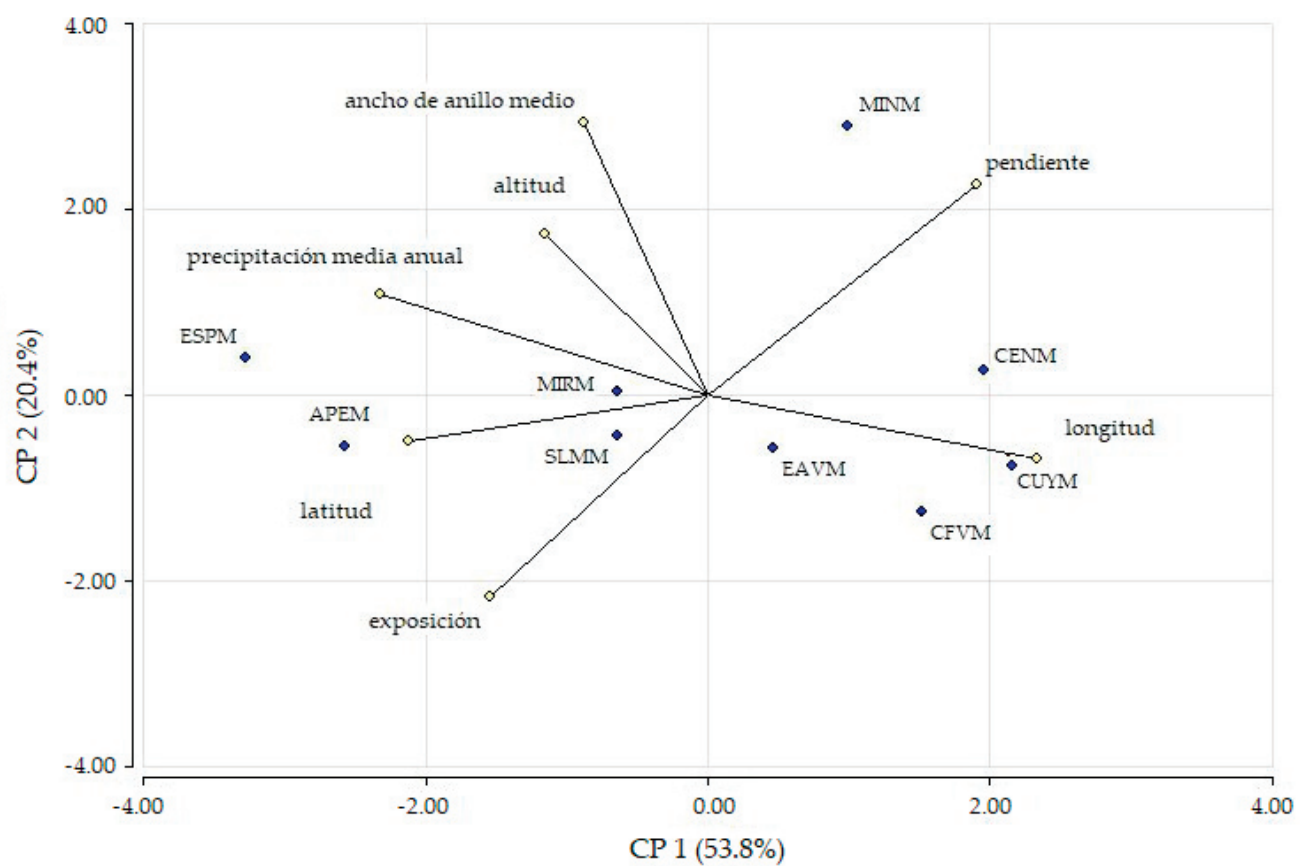

Figura 5. Análisis de componentes principales de cronologías residuales de individuos muertos de $A$. chilensis para nueve sitios de muestreo. Variables: altitud, pendiente, exposición, precipitación media anual, latitud y longitud, y ancho de anillo medio. Casos: sitios de muestreo (ver tabla 1 por el detalle de los códigos).

Figure 5. Principal component analysis of the residual chronologies of dead individuals of $A$. chilensis for nine sampling sites. Variables: altitude, slope, exposition, annual average rainfall, latitude and longitude, and average ring width. Cases: sampling sites (see table 1 for the detail of the codes).

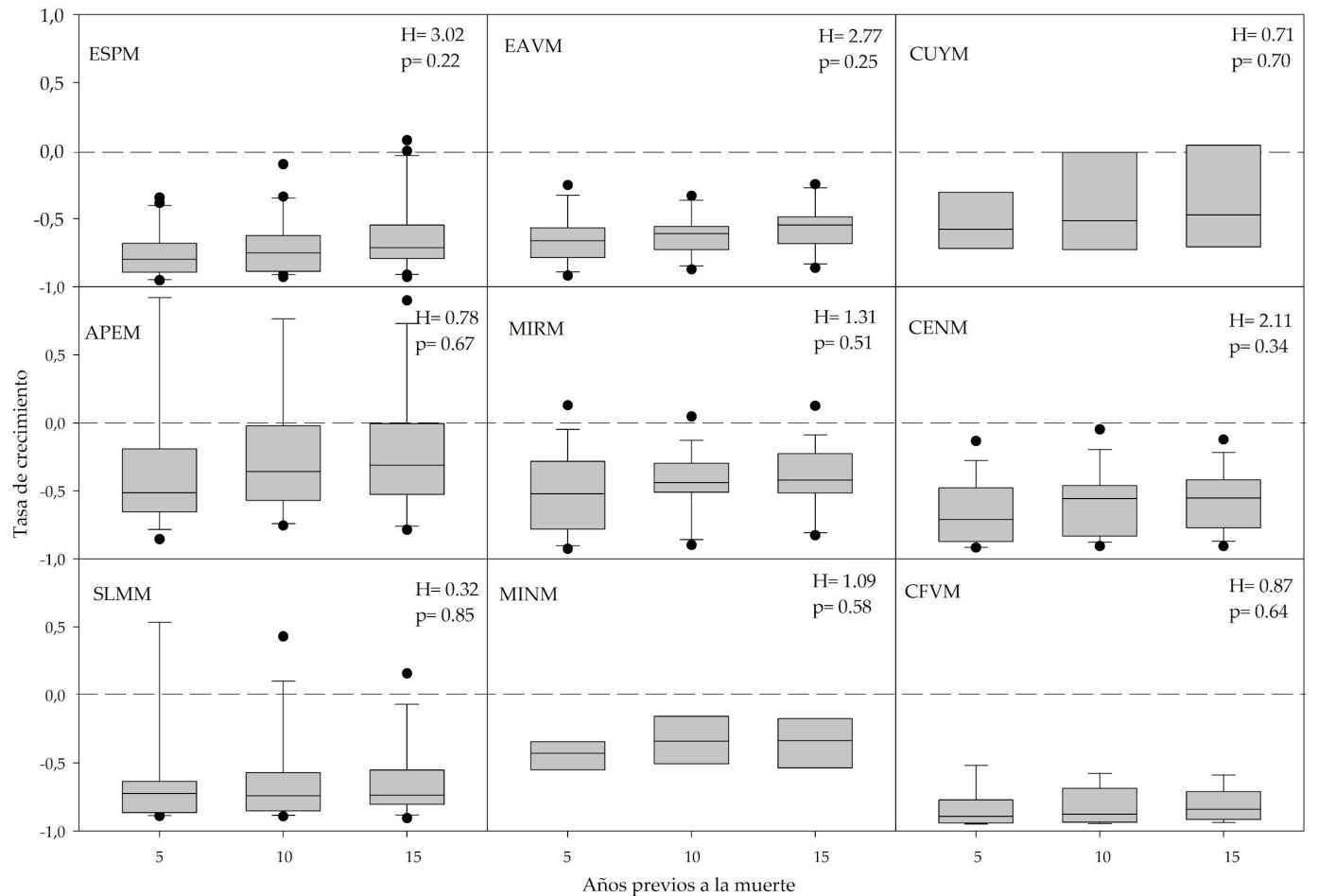

Figura 6. Tasas de crecimiento previas a la muerte para individuos de A. chilensis muertos, para diferentes ventanas temporales (5, 10, 15 años) En la parte superior derecha se indican los valores de los estadísticos de la prueba de Kruskal Wallis (ver Tabla 1 por el detalle de los códigos).

Figure 6. Growth rates prior to death for individuals of $A$. chilensis, for different temporal windows (5, 10, 15 years) In the upper right part, of the Kruskal Wallis test statistics are indicated (see Table 1 for the detail of the codes). 


\section{Patrones temporales de crecimiento}

El $74.2 \%$ de la variabilidad en los patrones de crecimiento de los individuos muertos fue explicado por los dos primeros componentes del ACP: componente principal 1 (CP1, $53.8 \%)$ y 2 (CP2, 20.4\%). Considerando el CP1, los sitios ESPM, APEM, MIRM, y SLMM (bosques mixtos y compactos) se relacionaron con valores negativos del eje y con las variables exposición, precipitación media anual, latitud, altitud y ancho de anillo. Por su parte, los sitios EAVM, CUYM, MINM, CFVM y CENM (bosques compactos y ecotonales) se relacionaron con valores positivos del eje y con las variables longitud y pendiente (Figura 5). Por su parte, el CP2 mostró que la variabilidad del ancho de anillo de los sitios ESPM, MIRM, MINM, y CENM se correlacionó con valores positivos del eje y con las variables ancho de anillo medio, precipitación media anual, altitud y pendiente, mientras que los sitios APEM, SLMM, EAVM, CFVM y CUYM se relacionaron con valores negativos del eje y con las variables latitud, longitud y exposición.

El análisis conjunto de las variables biofísicas y los patrones de crecimiento radial previos a la muerte de los individuos reflejó la posición y principales características de los sitios a lo largo del gradiente (Figura 5). Sin embargo, entre los dos grupos definidos por el CP1 sólo se encontraron diferencias significativas para las variables longitud $(\mathrm{H}=6, P=0.02)$ y precipitación $(\mathrm{H}=4.9, P=0.03)$

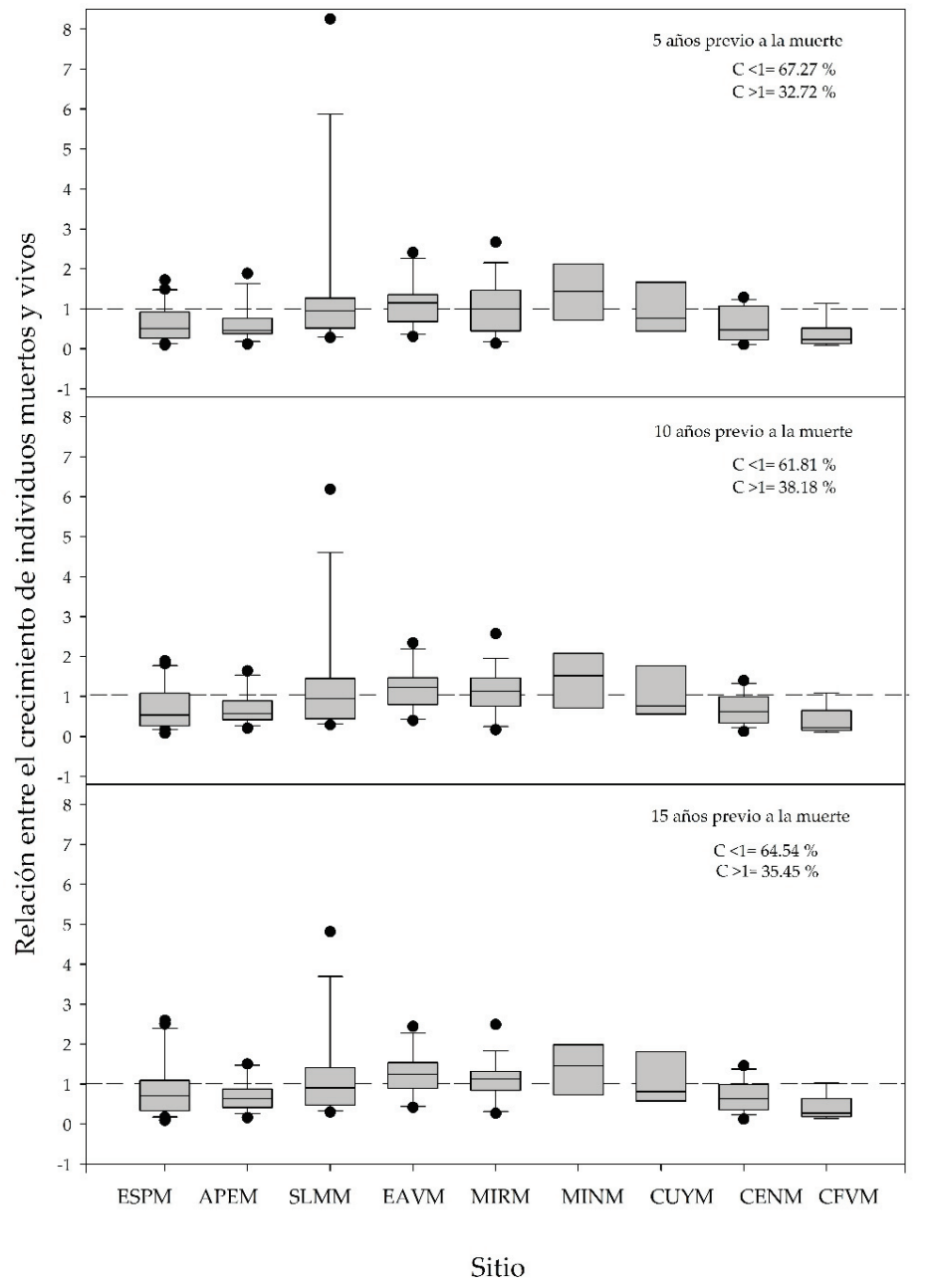

Figura 7. Relación entre el crecimiento previo a la muerte para distintas ventanas temporales y el crecimiento de individuos vivos para cada uno de los sitios de muestreo. $C=$ crecimiento; los porcentajes indican la cantidad de árboles cuyo crecimiento estuvo por encima o por debajo de 1 para todos los sitios en las distintas ventanas temporales (ver Tabla 1 por el detalle de los códigos).

Figure 7. Relationship between the growth prior to death for different temporal windows and the growth of live individuals for each of the sampling sites. $\mathrm{C}=$ growth; percentages indicate the number of trees whose growth was above or below 1 for all the sites in the different temporal windows (see Table 1 for the detail of the codes). 
(análisis no mostrados), variables íntimamente relacionadas con el gradiente ambiental. Los valores más altos de precipitación media anual se relacionaron con los sitios ESPM y APEM. La variable ancho de anillo fue mayor para los sitios SLMM y EAVM. A su vez, los sitios MINM y CENM presentaron mayor pendiente. Se observó que la pendiente aumenta con la longitud, así como la exposición con la altitud.

\section{Tasas de crecimiento previas a la muerte}

El análisis del crecimiento previo a la muerte dentro de cada sitio no arrojó diferencias significativas para las diferentes ventanas temporales estudiadas (5, 10 y 15 años) (Figura 6). En general, previo a la muerte los árboles crecieron menos con respecto al crecimiento medio de su vida (Figura 6). Asimismo, no se observaron diferencias significativas entre las tasas de crecimiento (ventanas de análisis) de los dos grupos ordenados según el ACP ( $\mathrm{H}=0.40, P=0.52$; análisis no mostrados).

De manera similar, la relación entre el crecimiento previo a la muerte de cada individuo y el crecimiento promedio de individuos vivos para el mismo sitio (Figura 7) no exhibió, en general, un patrón específico en el gradiente. Sin embargo, se observó que para las ventanas temporales estudiadas (5, 10 y 15 años), más del $60 \%$ de los individuos muertos presentaron crecimiento menor que los individuos vivos (relación $<1$ ). En particular, se observó que el $67.27 \%$, el $61.81 \%$ y el $64.4 \%$ de los individuos muertos crecieron menos que los vivos en los 5, 10 y 15 años previos a la muerte, respectivamente.

\section{DISCUSIÓN}

En este trabajo se estudiaron los patrones de crecimiento de individuos muertos de poblaciones de $A$. chilensis en sitios con distintas condiciones ambientales a lo largo del gradiente de precipitación donde la especie se distribuye. Los IMA de ancho de anillo presentaron diferencias significativas entre algunos bosques mixtos y compactos (ESPM, APEM, SLMM, MIRM y MINM), y bosques compactos y ecotonales (EAVM y CUYM). Este resultado coincide de forma parcial con trabajos pioneros en la especie, en los que sólo se reportaron crecimientos radiales menores en poblaciones de bosques ecotonales respecto de compactos y mixtos (Dezzoti and Sancholuz 1991). Aun así, las escasas diferencias llaman la atención si se tiene en cuenta que los sitios mésicos y húmedos (bosques compactos y mixtos) donde $A$. chilensis crece reciben entre 50 y 100\% más de precipitaciones, respectivamente, que aquellos situados en el extremo este del gradiente estudiado (Cordón et al. 1993). Para otras especies de los bosques Andino-Patagónicos, como el coihue (Nothofagus dombeyi), diferencias en los ambientes de los extremos del gradiente resultan, entre otras cosas, en diferentes tasas de crecimiento (Suárez and Kitzberger 2010).

Los patrones temporales de crecimiento previos a la muerte en individuos a lo largo del gradiente presentaron alta variabilidad temporal, parte de la cual fue común a la mayoría de los sitios. En ocho de los nueve sitios de estudio se observaron patrones similares de aumento del crecimiento previos al año 1900 y de disminuciones alrededor de los años 1900, 1922-23, 1943-44 y 1990. Sitios ubicados en el centro y oeste del gradiente de precipitación (ESPM, APEM, SLMM, EAVM, MIRM y MINM) y sitios en el extremo este del gradiente por otro (CUYM, CFVM y CENM) presentaron valores de correlación más altos entre sí, respectivamente. Esto da cuenta de una mayor señal común según la ubicación de los sitios en el gradiente, lo cual ya había sido reportado para las poblaciones marginales y compactas de la especie a lo largo de su distribución (Villalba and Veblen 1997). El ACP de las cronologías permitió identificar con claridad la existencia de dos grupos, lo que refuerza lo antes presentado. Por un lado, ordenó a sitios ubicados hacia el centro-este del gradiente (grupo de bosques compacto-ecotonal); por el otro, ordenó a los sitios ubicados hacia el oeste-centro (grupo de bosques mixto-compacto). A su vez, estos grupos están significativamente asociados a la precipitación y a la longitud, lo que remarca que las diferencias encontradas para estos grupos se explican por las diferencias pluviométricas antes discutidas. Sin embargo, y a pesar de que el resto de las variables biofísicas no se asociaron de manera significativa, el ACP preliminarmente permite evidenciar cierto grado de influencia de las condiciones biofísicas en el crecimiento de los sitios estudiados y los grupos resultantes del ordenamiento. La variabilidad del grupo mixto-compacto se asoció con altos valores de precipitación media anual, exposición y ancho de anillo y bajos valores de pendiente. Por su parte, el grupo compacto-ecotonal se asoció de manera positiva con la pendiente 
y negativa con el resto de las variables. Estos resultados sugieren que conjuntamente con la precipitación - principal determinante de la estructura y la fisonomía de estos bosquesalgunas variables biofísicas del sitio podrían condicionar los patrones de crecimiento y mortalidad a nivel local. En este sentido, se conoce el efecto que la exposición tiene sobre la demografía poblacional de la especie y el crecimiento de individuos de distinto sexo (Nuñez et al. 2008). A su vez, nuestros resultados están en la línea de lo reportado para otras especies de coníferas y especies tropicales, para las que la mortalidad no es explicada en su totalidad por las diferencias en la disponibilidad hídrica dentro de gradientes pluviométricos, sino, por ejemplo, por distintas condiciones topográficas, con diferencias en la mortalidad entre sitios bajos y planos, y sitios más altos y con pendiente (Zulueta 2016). Asimismo, la mortalidad en otras especies de coníferas fue explicada por diferencias en gradientes altitudinales y en las exposiciones de los sitios (Viguera Moreno 2012; González-Caro et al. 2016).

\section{Tasas de crecimiento previas a la muerte}

Considerando desde los 15 años previos a la muerte, los individuos presentaron menor crecimiento en comparación con el promedio del total de años que vivieron. De manera similar a nuestros resultados, la mayoría de los trabajos que estudiaron el proceso de mortalidad indican que, previo a este momento, los árboles presentan una disminución del crecimiento (Pedersen 1998; Bigler and Bugman 2004; Cailleret et al. 2016). Sin embargo, la ventana temporal de disminución tiene un amplio registro de 1 a 100 años (Cailleret et al. 2016) y, en otros casos, desde $<20$ años hasta $<5$ años, según los mecanismos que predisponen a la mortalidad (Berdanier and Clark 2016; Gessler et al. 2018). Según Mc Dowell y colaboradores (2008), el crecimiento radial reducido en los años previos a la muerte del árbol sería compatible con un agotamiento en sus reservas de carbono como consecuencia de un deterioro de la disponibilidad hídrica e incluso una mayor vulnerabilidad frente a los patógenos.

La relación entre el crecimiento previo a la muerte y el crecimiento medio de individuos vivos para el mismo sitio permitió conocer que en todos los sitios la mayoría de los individuos muertos $(>60 \%)$ crecieron menos que los individuos vivos. Esto concuerda con los resultados obtenidos en numerosos estudios en los que árboles en proceso de muerte mostraron menores tasas de crecimiento que individuos vivos (Pedersen 1998; Bigler and Bugmann 2004; Cailleret et al. 2016, 2017). Esta condición observada en todo el gradiente estudiado podría indicar una influencia de factores que actúan a mayor escala (e.g., climáticos), que, en combinación con las condiciones del sitio, resulten en una mayor vulnerabilidad de algunos individuos. Particularmente, dinámicas intra-sitio (como la competencia por agua en zonas limitadas por este recurso) se reportaron como influyentes en el proceso de mortalidad (Young et al. 2016).

Los patrones temporales de mortalidad, por su parte, resultaron muy variables, como ya fue reportado para la especie en estudios previos (Villalba and Veblen 1998; Amoroso and Larson 2010; Amoroso et al. 2015). Si bien no es del todo claro, se observan algunas diferencias entre sitios a lo largo del gradiente en relación con la frecuencia y la concentración de los eventos de mortalidad. Al igual que lo reportado para la especie (Villalba and Veblen 1998), sitios cercanos entre sí como ESPM y APEM (bosques mixtos), y, por otro lado, SLMM, EAVM y MIRM (bosques compactos), presentaron concordancia respecto de las fechas de mortalidad. Por otro lado, algunos sitios reportaron fechas con alta mortalidad que no se observan en otros sitios, como CUYM. Estas variaciones podrían estar asociadas con diferentes estrategias de la especie para hacer frente al estrés ambiental en relación a las condiciones ambientales en las que se desarrolla. Estas condiciones podrían involucrar la profundidad del suelo, la redistribución de nutrientes por las dinámicas intra-sitio, como así también la asignación de recursos a diferentes destinos como el crecimiento y el almacenamiento (Dietze et al. 2014). La falta de un patrón de mortalidad común a lo largo de la transecta fortalece la hipótesis de la influencia de las condiciones biofísicas del sitio sobre la dinámica y crecimiento en esta especie, sobre las cuales es necesario seguir indagando. Esto último podría contribuir a conocer cuáles son los mecanismos/características que permiten constituir los grupos mixto-compacto y compacto-ecotonal para poder entender mejor la respuesta de los árboles muertos de $A$. chilensis en diferentes condiciones ambientales. Sería importante que futuros estudios aborden este aspecto en mayor detalle en cuanto a los factores que influyen en él y también la relación de las fechas de ocurrencia de los 
eventos de mortalidad con la variabilidad climática, dado el conocido efecto que ésta tiene sobre la mortalidad en la especie (Villalba and Veblen 1998). En relación con esto último, y frente a las condiciones climáticas previstas para las próximas décadas, un aumento en la frecuencia e intensidad de las sequías podría incrementar los procesos de decaimiento y la consecuente muerte de los árboles (IPCC 2014). De este modo, la respuesta de los árboles a las sequías podría variar en el gradiente ambiental según las capacidades de resiliencia, recuperación y resistencia de las poblaciones frente a la sequía (Lloret et al. 2011). Estos parámetros comenzaron a ser evaluados para el área de estudio y podrían colaborar para determinar las similitudes en el ordenamiento de los dos grupos de árboles en el gradiente, particularmente en respuesta frente a los eventos hídricos extremos.

\section{Conclusiones}

Este trabajo representa el primer estudio que aborda la mortalidad episódica en bosques de Austrocedrus chilensis, incluyendo sitios a lo largo del gradiente de precipitación O-E sobre el que la especie se distribuye en la Argentina. El estudio de los patrones de crecimiento de los árboles muertos permitió determinar que el crecimiento radial promedio
(IMA) a lo largo del gradiente varía entre sitios. Además, la variabilidad temporal en el crecimiento mostró un ordenamiento en dos grupos: por un lado, poblaciones que incluyen bosques mixtos y compactos; por el otro, bosques compactos y ecotonales. Estas diferencias estarían reflejando principalmente las diferencias pluviométricas a lo largo del gradiente, aunque resulta factible también que condiciones biofísicas locales influyan en el crecimiento previo a la muerte de los individuos. En todos los sitios se registró que el crecimiento de los individuos previo a su muerte fue menor que en los individuos vivos (tasas negativas de crecimiento) para las mismas ventanas temporales, lo que indica un proceso de decaimiento para estos individuos. En este sentido, es fundamental realizar estudios futuros para avanzar sobre los procesos o mecanismos que conducen a los árboles a la muerte. En conclusión, estos resultados representan una contribución más amplia en lo que respecta a la caracterización de la variabilidad en los patrones de mortalidad a lo largo del gradiente ambiental donde crece A. chilensis. La relación entre los patrones de mortalidad y las condiciones ambientales es clave para la gestión y el diseño de estrategias de manejo y conservación de estos bosques frente a los futuros escenarios de cambio global.

\section{REFERENCIAS}

Adams, H. D., M. Guardiola-Claramonte, G. A. Barron-Gafford, J. C. Villegas, D. D. Breshears, C. B. Zou, and T. E. Huxman. 2009. Temperature sensitivity of drought-induced tree mortality portends increased regional die-off under global-change-type drought. Proceedings of the national academy of sciences 106:7063-7066. https://doi.org/10.1073/ pnas.0901438106.

Allen, C. D., and D. D. Breshears. 1998. Drought-induced shift of a forest-woodland ecotone: rapid landscape response to climate variation. Proceedings of the National Academy of Sciences 95:14839-14842. https://doi.org/10.1073/ pnas.95.25.14839.

Allen, C. D., A. K. Macalady, H. Chenchouni, D. Bachelet, M. McDowell, M. Vennetier, T. Kitzberger, D. Rigling Breshears, E. H. Hogg, P. González, R. Fensham, J. Castro, N. Demidova., J. Lim, G. Allard, R. Running, R. Semerci, and N. Cobb. 2010. A global overview of drought and heat-induced tree mortality reveals emerging climate change risks for forests. Forest Ecology and Management 259:660-684. https://doi.org/10.1016/j.foreco.2009.09.001.

Amoroso, M. M., and L. D. Daniels. 2010. Cambial mortality in declining Austrocedrus chilensis forests: implications for stand dynamics studies. Canadian Journal of Forest Research 40:885-893. https://doi.org/10.1139/X10-042.

Amoroso, M. M., and M. L. Suárez. 2015. La aplicación del análisis de los anillos de crecimiento a interrogantes ecológicos: Un breve repaso de la Dendrocronología en Hispanoamérica. Revista Ecosistemas 24:1-6. https://doi.org/ 10.7818/ECOS.2015.24-2.01.

Amoroso, M. M., L. D. Daniels, R. Villalba, and P. Cherubini. 2015. Does drought incite tree decline and death in Austrocedrus chilensis forests? Journal of vegetation science 26:1171-1183. https://doi.org/10.1111/jvs.12320.

Berdanier, A. B., and J. S. Clark. 2016. Multiyear drought-induced morbidity preceding tree death in southeastern US forests. Ecological Applications 26(1):17-23. https://doi.org/10.1890/15-0274.

Bianchi, E. 2016. Dinámica espacio-temporal de la relación entre el clima y el funcionamiento de los ecosistemas en Patagonia Norte. Doctor en Biología. Universidad Nacional del Comahue. Neuquén, Argentina.

Bigler, C., and H. Bugmann. 2004. Predicting the time of tree death using dendrochronological data. Ecological Applications 14:902-914. https://doi.org/10.1890/03-5011.

Bigler, C., D. G. Gavin, C. Gunning, and T. T. Veblen. 2007. Drought induces lagged tree mortality in a subalpine forest in the Rocky Mountains. Oikos 116(12):1983-1994. https://doi.org/10.1111/j.2007.0030-1299.16034.x. 
Bran, D., A. Pérez, D. Barrios, M. Pastorino, and J. Ayesa. 2002. Eco- Región Valdiviana: distribución actual de los bosques de "ciprés de la cordillera" (Austrocedrus chilensis). INTA, APN, FVSA. Informe Preliminar, Bariloche, Argentina. Pp. 12.

Bunn, A. G. 2008. A dendrochronology program library in R (dplR). Dendrochronologia 26:115-124. https://doi.org/ 10.1016/j.dendro.2008.01.002.

Cailleret, M., C. Bigler, and H. Bugmann. 2016. Towards a common methodology for developing logistic tree mortality models based on ring-width data. Ecological Applications 26:1827-1841. https://doi.org/10.1890/15-1402.1.

Cailleret, M., S. Jansen, E. M. Robert, L. Desoto, T. Aakala, J. A. Antos, and V. Čada. 2017. A synthesis of radial growth patterns preceding tree mortality. Global Change Biology 23(4):1675-1690. https://doi.org/10.1111/gcb.13535.

Camarero, J. J., A. Q. Sangüesa-Barreda, E. Alla, M. González de Andrés, S. M. Maestro Martínez, and S. M. VicenteSerrano. 2012. Los precedentes y las respuestas de los árboles a sequías extremas revelan los procesos involucrados en el decaimiento de bosques mediterráneos de coníferas. Ecosistemas 21:22-30. https://doi.org/10.7818/ECOS.2012.213.04 .

Cordón, V., J. Forquera, and J. Gastiazoro. 1993. Estudio microclimático del Área Cordillerana del Sudoeste de la provincia de Río Negro. Cartas de Precipitación. Universidad Nacional del Comahue. Facultad de Ciencias Agrarias. Cinco Saltos. Río Negro. Argentina. Pp. 17.

Dezzotti, A., and L. Sancholuz. 1991. Los bosques de Austrocedrus chilensis en Argentina: ubicación, estructura y crecimiento. Bosque 12:43-52. https://doi.org/10.4206/bosque.1991.v12n2-04.

Dietze, M. C., A. Sala, M. S. Carbone, C. I. Czimczik, J. A. Mantooth, A. D. Richardson, and R. Vargas. 2014. Nonstructural carbon in woody plants. Annual Review of Plant Biology 65:667-687. https://doi.org/10.1146/annurev-arplant-050213040054 .

Gallo, L. A., M. J. Pastorino, C. Donoso, A. Premoli, and R. Ipinza. 2004. Variación en Austrocedrus chilensis (D. Don) Pic. Ser et Bizzarri (Ciprés de la Cordillera). Variación intraespecífica en las especies arbóreas de los bosques templados de Chile y Argentina.

García-González, I., E. Díaz-Vizcaino, and A. Martínez-Cortizas. 1997. Evidence for a common and a species-specific climatic response of Oak and Birch on a Northern Galician site (NW SPAIN) by means of multivariate procedures. Dendrochronologia 15:119-127.

Garreaud, R. D., M. Vuille, R. Compagnucci, and J. Marengo. 2009. Present-day South American climate. Palaeogeog raphy, Palaeoclimatology, Palaeoecology 281:180-195. https://doi.org/10.1016/j.palaeo.2007.10.032.

Gessler, A., M. Cailleret, J. Joseph, L. Schönbeck, M. Schaub, M. Lehmann, K. Treydte, A. Rigling, G. Timofeeva, and M. Saurer. 2018. Drought induced tree mortality-a tree-ring isotope based conceptual model to assess mechanisms and predispositions. New Phytologist 219(2):485-490. https://doi.org/10.1111/nph.15154.

González, M. E., M. M. Amoroso, A. Lara, T. T. Veblen, C. Donoso, T. Kitzberger, I. Mundo, A. Holz, A. Casteller, J. Paritsis, A. Muñoz, M. L. Suárez, and A. Promis. 2014. Ecología de disturbios y su influencia en los ecosistemas forestales templados de Chile y Argentina. En C. Donoso. M. E. González, A. Lara and P. Donoso (eds.). Ecología Forestal: Bases para el Manejo Sustentable de los Bosques Nativos. Marisa Cuneo Editores. Valdivia.

Grissino-Mayer, H. 2001. FHX2-Software for analysing temporal and spatial patterns in fire regimes from tree rings. Tree-Ring Research 57:115-124.

Holmes, R. 1983. Computer-assisted quality control in tree-ring dating and measurement. Tree-Ring Bulletin 43:6975.

IPCC. 2014. Climate Change 2014: Synthesis Report. Contribution of Working Groups I, II and III to the Fifth Assessment Report of the Intergovernmental Panel on Climate Change. Core Writing Team, R. K. Pachauri and L. A. Meyer (eds.). IPCC, Geneva, Switzerland. Pp. 151.

Kitzberger, T., T. T. Veblen, and R. Villalba. 2000. Métodos dendroecológicos y sus aplicaciones en estudios de dinámica de bosques templados de Sudamérica. Dendrocronología en América Latina. Pp. 17-78.

La Manna, L. 2005. Caracterización de los suelos bajo bosque de Austrocedrus chilensis a través de un gradiente climático y topográfico en Chubut, Argentina. Nota técnica. Bosque 26:137-153. https://doi.org/10.4067/S071792002005000200017.

Lloret, F., Keeling, E. G., and A. Sala. 2011. Components of tree resilience: effects of successive low-growth episodes in old ponderosa pine forests. Oikos 120(12):1909-1920. https://doi.org/10.1111/j.1600-0706.2011.19372.x.

Matías, L. 2012. Cambios en los límites de distribución de especies arbóreas como consecuencia de las variaciones climáticas. Revista Ecosistemas 21:91-96. https://doi.org/10.7818/ECOS.2012.21-3.12.

McDowell, N., W. T. Pockman, C. D. Allen, D. D. Breshears, N. Cobb, T. Kolb, and E. Yepez. 2008. Mechanisms of plant survival and mortality during drought: why do some plants survive while others succumb to drought? New Phytologist 178:719-739. https://doi.org/10.1111/j.1469-8137.2008.02436.x.

McKenney, D. W, J. H. Pedlar, K. Lawrence, K. Campbell, and M. F. Hutchinson. 2007. Potential impacts of climate change on the distribution of North American trees. AIBS Bulletin 57:939-948. https://doi.org/10.1641/B571106.

Mundo, I. 2011. Historia de incendios en bosques de Araucaria araucana (Molina) K. Koch de Argentina a través de un análisis dendroecológico. Doctor en Ciencias Naturales. Universidad Nacional de La Plata, Argentina.

Nuñez, C. I., M. A. Nuñez, and T. Kitzberger. 2008. Sex-related spatial segregation and growth in a dioecious conifer along environmental gradients in northwestern Patagonia. Ecoscience 15:73-80. https://doi.org/10.2980/11956860(2008)15[73:SSSAGI]2.0.CO;2.

Pastorino, M. J., and L. A. Gallo. 2002. Quaternary evolutionary history of Austrocedrus chilensis, a cypress native to the 
Andean-Patagonian forest. Journal of Biogeography 29:1167-1178.

Pastorino, M. J., M. M. Fariña, D. Bran, and L. A. Gallo. 2006. Extremos geográficos de la distribución natural de Austrocedrus chilensis (Cupressaceae). Bol Soc Argent Bot 41:307-311.

Pastorino, M., A. Aparicio, and M. M. Azpilicueta. 2015. Regiones de Procedencia del Ciprés de la Cordillera y bases conceptuales para el manejo de sus recursos genéticos en Argentina. Ediciones INTA, Buenos Aires.

Pedersen, B. S. 1998. Modeling tree mortality in response to short-and long-term environmental stresses. Ecological Modelling 105:347-351. https://doi.org/10.1016/S0304-3800(97)00162-2.

Ruiz-Benito, P., A. Herrero, and M. A. Zavala. 2013. Vulnerabilidad de los bosques españoles frente al Cambio Climático: evaluación mediante modelos. Ecosistemas 22:21-28. https://doi.org/10.7818/ECOS.2013.22-3.04.

Rodríguez Catón, M. 2014. Influencia de las variaciones climáticas en el decaimiento de bosques de Nothofagus pumilio (Lenga) en el norte de la Patagonia. Doctora en Biología. Universidad Nacional de Cuyo, Mendoza, Argentina.

Stokes, M. A., and T. L. Smiley. 1968. An introduction to tree-ring dating. University of Arizona Press, Tucson, Arizona, USA.

Suárez, M. L., and T. Kitzberger. 2008. Recruitment patterns following a severe drought: long-term compositional shifts in Patagonian forests. Can J For Res 38:3002-3010. https://doi.org/10.1139/X08-149.

Suárez, M. L., and T. Kitzberger. 2010. Differential effects of climate variability on forest dynamics along a precipitation gradient in northern Patagonia. Journal of Ecology 98:1023-1034. https://doi.org/10.1111/j.1365-2745.2010.01698.x.

Suárez, M. L., and M. M. Amoroso. 2013. Bosques y cambio climático. Parte II: Eventos de mortalidad en bosques y su relación con la ocurrencia de sequías. INTA. Cuadernillo $\mathrm{N}^{\circ} 4$.

Villalba, R., and T. T. Veblen. 1996. A tree-ring record of dry spring-wet summer events in the forest-steppe ecotone, northern Patagonia, Argentina. Tree Rings, Environment and Humanity. Pp. 107-116.

Villalba, R., and T. T. Veblen. 1997. Regional patterns of tree population age structures in Northern Patagonia: Climatic and Disturbance Influences. Journal of Ecology 85:113-124. https://doi.org/10.2307/2960643.

Villalba, R., and T. T. Veblen. 1998. Influences of large-scale climatic variability on episodic tree mortality in northern Patagonia. Ecology 79:2624-2640. https://doi.org/10.1890/0012-9658(1998)079[2624:IOLSCV]2.0.CO;2.

Young, D. J., J. T. Stevens, J. M. Earles, J. Moore, A. Ellis, A. L. Jirka, and A. M. Latimer. 2017. Long-term climate and competition explain forest mortality patterns under extreme drought. Ecology Letters 20(1):78-86. https://doi.org/ 10.1111/ele.12711.

Zulueta, D. 2016. Simp, L. F. O. D. V. Bosques y Cambio Global. Colombia Forestal 19(1):9-12. 